\title{
Ultradilute Low-Dimensional Liquids
}

\author{
D. S. Petrov ${ }^{1}$ and G. E. Astrakharchik ${ }^{2}$ \\ ${ }^{1}$ LPTMS, CNRS, Univ. Paris Sud, Université Paris-Saclay, 91405 Orsay, France \\ ${ }^{2}$ Departament de Física, Campus Nord B4-B5, Universitat Politècnica de Catalunya, E-08034 Barcelona, Spain
}

(Received 24 May 2016; revised manuscript received 28 July 2016; published 1 September 2016)

\begin{abstract}
We calculate the energy of one- and two-dimensional weakly interacting Bose-Bose mixtures analytically in the Bogoliubov approximation and by using the diffusion Monte Carlo technique. We show that in the case of attractive inter- and repulsive intraspecies interactions the energy per particle has a minimum at a finite density corresponding to a liquid state. We derive the Gross-Pitaevskii equation to describe droplets of such liquids and solve it analytically in the one-dimensional case.
\end{abstract}

DOI: 10.1103/PhysRevLett.117.100401

According to van der Waals' theory the fundamental property of a liquid to form self-bound states with free surface is due to the shape of the interaction potential which typically has a repulsive core and a more extended attractive part. Usual liquids are dense and almost incompressible since particles prefer to be at the potential minima. A qualitatively different type of liquid, dilute one, has very recently been observed in a Bose-condensed Dy gas characterized by anisotropic dipolar interactions [1,2] and a similar phenomenon has been predicted to occur in three-dimensional Bose-Bose mixtures with isotropic contact interactions [3]. In both cases the system, collapsing from the mean-field viewpoint, is stabilized by quantum many-body effects; each particle feels the attractive meanfield interaction proportional to the density $n$ compensated by the positive Lee-Huang-Yang correction $\propto n^{3 / 2}$ [1-5]. Such liquids and their finite-size droplets remain dilute and weakly interacting allowing for a well-controlled perturbative description. They also have quite peculiar features: their very existence is a direct manifestation of beyond mean-field effects, they require no trapping and their bulk density and shape are tunable by changing interactions, in the absence of external trapping they can reach zero temperature by evaporation, etc.

In this Letter, motivated by the enhanced role of beyondmean-field effects in low dimensions [6], we consider twoand one-dimensional Bose-Bose mixtures and show that with decreasing the dimensionality the liquid phase not only persists, but becomes more ubiquitous and remarkable. We find that in the two-dimensional case the energy per particle is proportional to $n\left[\ln \left(n / n_{0}\right)-1\right]\left(n_{0}\right.$ is the equilibrium density), the liquid state exists as long as the interspecies interaction is weakly attractive and the intraspecies ones are weakly repulsive. This contrasts the threedimensional case where a critical interspecies attraction is needed to liquefy the mixture. Interestingly, we find that a three-dimensional mixture in the gas phase can become liquid if confined to the quasi-two-dimensional geometry. In the one-dimensional case the liquid phase originates from the competition of a repulsive mean-field term $\propto n$ and attractive beyond mean-field correction $\propto-n^{1 / 2}$. Counterintuitively, this means that a one-dimensional mixture, stable from the mean-field viewpoint, is actually unstable towards the formation of a liquid droplet. We analytically describe its shape and other properties.

Consider two equal-mass bosonic species $(\sigma=\uparrow ; \downarrow)$, with densities $n_{\uparrow}$ and $n_{\downarrow}$, governed by the Hamiltonian

$$
\begin{aligned}
H= & \sum_{\sigma, \mathbf{k}} \frac{k^{2}}{2} \hat{a}_{\sigma, \mathbf{k}}^{\dagger} \hat{a}_{\sigma, \mathbf{k}} \\
& +\frac{1}{2} \sum_{\sigma, \sigma^{\prime}, \mathbf{k}_{1}, \mathbf{k}_{2}, \mathbf{q}} \hat{a}_{\sigma, \mathbf{k}_{1}+\mathbf{q}}^{\dagger} \hat{a}_{\sigma^{\prime}, \mathbf{k}_{2}-\mathbf{q}}^{\dagger} U_{\sigma \sigma^{\prime}}(\mathbf{q}) \hat{a}_{\sigma, \mathbf{k}_{1}} \hat{a}_{\sigma^{\prime}, \mathbf{k}_{2}},
\end{aligned}
$$

where $U_{\sigma \sigma^{\prime}}$ are short-range interaction potentials and we set $m=\hbar=1$. As usual $[7,9,10]$, one substitutes $U_{\sigma \sigma^{\prime}}$ by effective potentials, characterized by the same scattering amplitudes for relevant collision energies but more suitable for perturbative expansions.

We first discuss the two-dimensional case and take $U_{\sigma \sigma^{\prime}}(\mathbf{q})=g_{\sigma \sigma^{\prime}}=$ const $\ll 1$ for $|\mathbf{q}|<\kappa$ and $U_{\sigma \sigma^{\prime}}(\mathbf{q})=0$ for $|\mathbf{q}|>\kappa$. The coupling constants $g_{\sigma \sigma^{\prime}}$ and the cutoff $\kappa$ are related to the two-dimensional scattering lengths $a_{\sigma \sigma^{\prime}}>0$ by $g_{\sigma \sigma^{\prime}}=4 \pi / \ln \left(\epsilon_{\sigma \sigma^{\prime}} / \kappa^{2}\right)$, where $\epsilon_{\sigma \sigma^{\prime}}=4 e^{-2 \gamma} / a_{\sigma \sigma^{\prime}}^{2}$ and $\gamma$ is Euler's constant. This relation ensures that at low energy, $z \ll \kappa^{2}$, the scattering $t$ matrix behaves as $t_{\sigma \sigma^{\prime}}(z) \approx$ $4 \pi / \ln \left(-\epsilon_{\sigma \sigma^{\prime}} / z\right)[7,11]$ consistent with the Born series expansion $\quad t_{\sigma \sigma^{\prime}}(z) \approx g_{\sigma \sigma^{\prime}}\left[1-g_{\sigma \sigma^{\prime}} \ln \left(-\kappa^{2} / z\right) / 4 \pi+\cdots\right]$. One can see that the perturbation series in terms of $\left|t_{\sigma \sigma^{\prime}}\right| \ll$ 1 and $\left|g_{\sigma \sigma^{\prime}}\right| \ll 1$ are equivalent as long as $\kappa^{2}$ is larger but not exponentially larger than the typical interaction energy $z$ which is the product of the density $n$ and the $t$ matrix (with the logarithmic accuracy one can simply use $z \sim n$ ). An appropriate value of $\kappa$ can always be found in the weaklyinteracting regime where the scattering lengths are exponentially small (repulsion) or large (attraction) compared to the mean interparticle separation. 
In order to calculate the ground-state energy of the mixture up to second order terms in $g$ we do the standard Bogoliubov theory (see, for example, Ref. [10]). Namely, we assume a macroscopic condensate population $\hat{a}_{\sigma, 0} \approx \sqrt{n_{\sigma}}$, expand (1) up to bilinear terms in the operators $\hat{a}_{\sigma, \mathbf{k}}^{\dagger}, \hat{a}_{\sigma, \mathbf{k}}$ for $k \neq 0$, and diagonalize the bilinear form arriving at the ground-state energy density

$E=\frac{1}{2} \sum_{\sigma \sigma^{\prime}} g_{\sigma \sigma^{\prime}} n_{\sigma} n_{\sigma^{\prime}}+\frac{1}{2} \sum_{ \pm} \sum_{|\mathbf{k}|<\kappa}\left[E_{ \pm}(k)-k^{2} / 2-c_{ \pm}^{2}\right]$,

where $E_{ \pm}(k)=\sqrt{c_{ \pm}^{2} k^{2}+k^{4} / 4}$ are the Bogoliubov modes with sound velocities $c_{ \pm}$defined by

$c_{ \pm}^{2}=\frac{g_{\uparrow \uparrow} n_{\uparrow}+g_{\downarrow \downarrow} n_{\downarrow} \pm \sqrt{\left(g_{\uparrow \uparrow} n_{\uparrow}-g_{\downarrow \downarrow} n_{\downarrow}\right)^{2}+4 g_{\uparrow \downarrow}^{2} n_{\uparrow} n_{\downarrow}}}{2}$.

The momentum integration in Eq. (2) gives

$$
E_{2 \mathrm{D}}=\frac{1}{2} \sum_{\sigma \sigma^{\prime}} g_{\sigma \sigma^{\prime}} n_{\sigma} n_{\sigma^{\prime}}+\frac{1}{8 \pi} \sum_{ \pm} c_{ \pm}^{4} \ln \frac{c_{ \pm}^{2} \sqrt{e}}{\kappa^{2}}
$$

Recalling that $g_{\sigma \sigma^{\prime}}=4 \pi / \ln \left(\epsilon_{\sigma \sigma^{\prime}} / \kappa^{2}\right)$ one can check that to the chosen order $\partial E_{2 \mathrm{D}} / \partial \kappa^{2}=0$, i.e., the final result (4) depends only on $n_{\sigma}, a_{\sigma \sigma^{\prime}}$, and not on $\kappa$.

We now turn to the interesting for us case $1 / a_{\uparrow \downarrow} \ll$ $\left\{\sqrt{n_{\uparrow}}, \sqrt{n_{\downarrow}}\right\} \ll\left\{1 / a_{\uparrow \uparrow}, 1 / a_{\downarrow \downarrow}\right\}$ where the interspecies interaction is weakly attractive and intraspecies ones are weakly repulsive. Let us introduce an auxiliary energy parameter $\Delta$ and a new set of coupling constants defined by $\tilde{g}_{\sigma \sigma^{\prime}}=4 \pi / \ln \left(\epsilon_{\sigma \sigma^{\prime}} / \Delta\right)$. We choose $\Delta$ such that $\tilde{g}_{\uparrow \downarrow}^{2}=\tilde{g}_{\uparrow \uparrow} \tilde{g}_{\downarrow \downarrow} \quad$ or, $\quad$ explicitly, $\quad \Delta=\sqrt{\epsilon_{\uparrow \downarrow} \sqrt{\epsilon_{\uparrow \uparrow} \epsilon_{\downarrow \downarrow}}} \times$ $\exp \left[-\ln ^{2}\left(\epsilon_{\uparrow \uparrow} / \epsilon_{\downarrow \downarrow}\right) / 4 \ln \left(\epsilon_{\uparrow \uparrow} \epsilon_{\downarrow \downarrow} / \epsilon_{\uparrow \downarrow}^{2}\right)\right]$. Then we substitute the expansion $g_{\sigma \sigma^{\prime}} \approx \tilde{g}_{\sigma \sigma^{\prime}}\left[1+\tilde{g}_{\sigma \sigma^{\prime}} \ln \left(\kappa^{2} / \Delta\right) / 4 \pi+\cdots\right]$ into Eq. (4) and keep terms up to second order in the new small parameters $\tilde{g}_{\sigma \sigma^{\prime}}$. The energy density then reads

$$
\begin{aligned}
E_{2 \mathrm{D}}= & \frac{1}{2}\left(\tilde{g}_{\uparrow \uparrow}^{1 / 2} n_{\uparrow}-\tilde{g}_{\downarrow \downarrow}^{1 / 2} n_{\downarrow}\right)^{2} \\
& +\frac{1}{8 \pi}\left(\tilde{g}_{\uparrow \uparrow} n_{\uparrow}+\tilde{g}_{\downarrow \downarrow} n_{\downarrow}\right)^{2} \ln \frac{\left(\tilde{g}_{\uparrow \uparrow} n_{\uparrow}+\tilde{g}_{\downarrow \downarrow} n_{\downarrow}\right) \sqrt{e}}{\Delta} .
\end{aligned}
$$

Properties of the liquid phase in free space are obtained by minimizing the grand potential density $E_{2 \mathrm{D}}-\mu_{\uparrow} n_{\uparrow}-\mu_{\downarrow} n_{\downarrow}$ and by requiring that its value be zero (zero pressure). Explicitly, $E_{2 \mathrm{D}}-\sum_{\sigma}\left(\partial E_{2 \mathrm{D}} / \partial n_{\sigma}\right) n_{\sigma}=0$. One can show that possible values of $n_{\uparrow}$ and $n_{\downarrow}$ are close to the line $n_{\uparrow} / n_{\downarrow}=\sqrt{\tilde{g}_{\downarrow \downarrow} / \tilde{g}_{\uparrow \uparrow}}$ where the dominant first-order term in Eq. (5) vanishes. Particularly, for $n=n_{\uparrow}=n_{\downarrow} \sqrt{\tilde{g}_{\downarrow \downarrow} / \tilde{g}_{\uparrow \uparrow}}$ Eq. (5) reduces to the form $\propto \tilde{g}^{2} n^{2}\left[\ln \left(n / n_{0}\right)-1\right]$, where $n_{0} \sim \Delta /|\tilde{g}|$ is the equilibrium density at which the grand potential vanishes or, equivalently, the energy per particle $\propto E_{2 \mathrm{D}} / n$ reaches its minimum as a function of $n$. We a posteriori verify that $\kappa^{2} / \Delta$ is not exponentially large and, therefore, the small parameters $g$ and $\tilde{g}$ are equivalent.

In the symmetric case $a_{\uparrow \uparrow}=a_{\downarrow \downarrow}=a$ and $n_{\uparrow}=n_{\downarrow}=n$, one has $\Delta=\sqrt{\epsilon_{\uparrow \downarrow} \epsilon_{\uparrow \uparrow}}$, the energy density simplifies to

$$
E_{2 \mathrm{D}}=\frac{8 \pi n^{2}}{\ln ^{2}\left(a_{\uparrow \downarrow} / a\right)}\left[\ln \left(n / n_{0}\right)-1\right],
$$

and the equilibrium density of each component reads

$$
n_{0}=\frac{e^{-2 \gamma-3 / 2} \ln \left(a_{\uparrow \downarrow} / a\right)}{2 \pi} .
$$

The knowledge of the equation of state (6) permits us to find the spinodal point. Defined by the condition $\partial^{2} E_{2 \mathrm{D}} / \partial n^{2}=0$ it is located at $n=e^{-1 / 2} n_{0} \approx 0.61 n_{0}$. The mixture is thus metastable for $0.61 n_{0}<n<n_{0}$. Note that since $1 / \ln \left(a_{\uparrow \downarrow} / a\right) \ll 1$ the parameter $n a^{2} \propto$ $\left(a / a_{\uparrow \downarrow}\right) \ln \left(a_{\uparrow \downarrow} / a\right)$ is exponentially small. We are thus dealing with an extremely dilute liquid qualitatively different from usual liquids where $n a^{2} \sim 1$.

In order to check the universality of our theory and importance of higher-order corrections we perform diffusion Monte Carlo (DMC) calculations for the symmetric mixture $\left(n=n_{\uparrow}=n_{\downarrow}\right.$ and $\left.a=a_{\uparrow \uparrow}=a_{\downarrow \downarrow}\right)$ for various densities and interaction potentials. The DMC method gives the ground-state energy exactly and it has been applied to the one-component two-dimensional Bose gas in Ref. [12]. In our case the convergence is enhanced by using the guiding wave function in the Jastrow pair-product form where we match the inter- and intraspecies two-body scattering solutions at short distances with the long-range phononic behavior at large distances [13]. The calculations are performed in a finite box with periodic boundary conditions and the results are extrapolated to the thermodynamic limit $[14,15]$. In Fig. 1 we present the density dependence of the energy per particle. As expected, with decreasing $1 / \ln \left(a_{\uparrow \downarrow} / a\right)$ the numerical results converge toward our theory and the rate of this convergence is consistent with the scaling $n^{2} / \ln ^{3}\left(a_{\uparrow \downarrow} / a\right)$ for the nextorder correction to Eq. (6).

Let us now comment on the applicability of the above results to quasi-two-dimensional atomic mixtures. The passage from three-dimensional scattering parameters to two-dimensional ones is realized by using the formula $\epsilon_{\sigma \sigma^{\prime}}=\left(B / \pi l_{0}^{2}\right) \exp \left[\sqrt{2 \pi} l_{0} / a_{\sigma \sigma^{\prime}}^{(3 \mathrm{D})}\right][16]$, where $a_{\sigma \sigma^{\prime}}^{(3 \mathrm{D})}$ are the three-dimensional scattering lengths, $l_{0}$ is the oscillator length in the confinement direction, and $B \approx 0.9$. In particular, in the symmetric case, $a_{\uparrow \uparrow}^{(3 \mathrm{D})}=a_{\downarrow \downarrow}^{(3 \mathrm{D})}=a^{(3 \mathrm{D})}$, the equilibrium densities of the components equal 


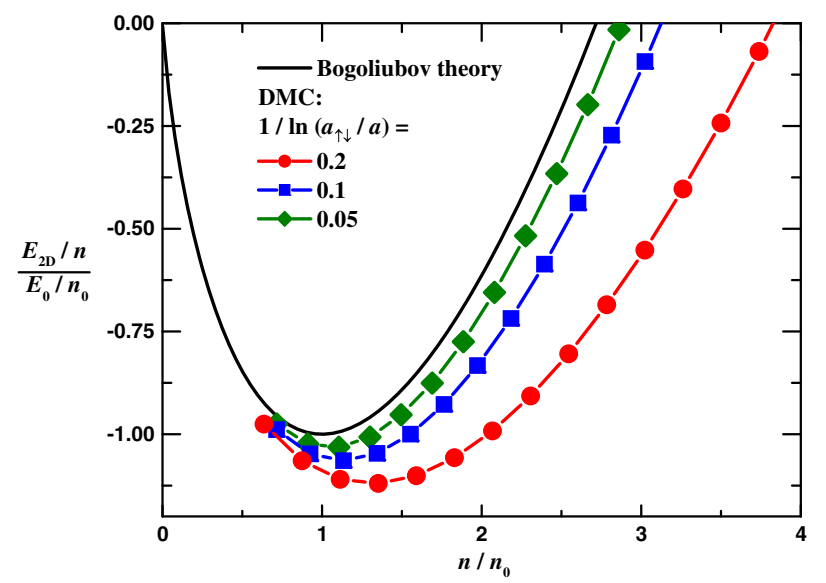

FIG. 1. The energy per particle $E_{2 \mathrm{D}} / 2 n$ versus $n$ for the twodimensional mixture with $a_{\uparrow \uparrow}=a_{\downarrow \downarrow}=a$ and $n_{\uparrow}=n_{\downarrow}=n$. We rescale the vertical and horizontal axes, respectively, by $E_{0} / 2 n_{0}=\left|E_{2 \mathrm{D}}\left(n_{0}\right)\right| / 2 n_{0}$ and $n_{0}$ calculated in the Bogoliubov approximation [Eqs. (6)-(7)]. The solid black line is the result of Eq. (6) and the scattered data are the DMC results for $1 / \ln \left(a_{\uparrow \downarrow} / a\right)=0.2$ (red, circles), 0.1 (blue, squares), and 0.05 (green, diamonds) corresponding to $n_{0} a^{2}=3.8 \times 10^{-4}$, $5.1 \times 10^{-6}$, and $4.6 \times 10^{-10}$, respectively. The interspecies (intraspecies) interactions are modeled by square wells (soft disks) with the range $R_{0}$ fixed by $n R_{0}^{2}=5 \times 10^{-3}$ and with the depths (heights) adjusted in order to obtain the desired values of $a_{\sigma \sigma^{\prime}}$. As a universality check we have significantly reduced $R_{0}$ and found that the energy changes at most by the symbol size.

$$
n_{0}=\frac{B\left[1 / a^{(3 \mathrm{D})}-1 / a_{\uparrow \downarrow}^{(3 \mathrm{D})}\right]}{4(2 \pi e)^{3 / 2} l_{0}} e^{\sqrt{\pi / 2}\left[l_{0} / a_{\uparrow \downarrow}^{(3 \mathrm{D})}+l_{0} / a^{(3 \mathrm{D})}\right]} .
$$

The weakly interacting regime in this case is ensured by the inequality $\quad \ln \left(a_{\uparrow \downarrow} / a\right)=\sqrt{\pi / 2}\left[l_{0} / a^{(3 \mathrm{D})}-l_{0} / a_{\uparrow \downarrow}^{(3 \mathrm{D})}\right] \gg 1$ and the requirement that typical transverse energies be much smaller than $1 / l_{0}^{2}$ (two-dimensional regime) practically reduces to $-\left[l_{0} / a_{\uparrow \downarrow}^{(3 \mathrm{D})}+l_{0} / a^{(3 \mathrm{D})}\right] \gg 1$. We can rewrite these two conditions as $0<-a_{\uparrow \downarrow}^{(3 \mathrm{D})}<a^{(3 \mathrm{D})} \ll l_{0}$. Note that a three-dimensional mixture satisfying $0<$ $-a_{\uparrow \downarrow}^{(3 \mathrm{D})}<a^{(3 \mathrm{D})}$ is in the stable gas phase since the interspecies attraction is too weak. We thus find a curious fact that by introducing the confinement the mixture becomes liquid. The nonsymmetric case is analysed in the same fashion and we finally note that suitable combinations of $a_{\sigma \sigma^{\prime}}^{(3 \mathrm{D})}$ are available for hyperfine components $F=1, m_{F}=$ -1 and $F=1, m_{F}=0$ of ${ }^{39} \mathrm{~K}[17,18]$.

Let us now discuss finite-size droplets of the liquid. The derivation of the corresponding Gross-Pitaevskii equation follows the same path as in the three-dimensional case [3]. In short, the length scale on which the droplet profile changes is of order $\xi \sim 1 / \sqrt{|\mu|}$, where the chemical potential $\mu \sim$ $-n / \ln ^{2}\left(a_{\uparrow \downarrow} / a\right)$ [see Eq. (6)]. On the other hand, excitations mostly contributing to the second-order terms in Eqs. (2) and (4) belong to the upper Bogoliubov branch and have wavelengths $\sim 1 / c_{+} \propto 1 / \sqrt{n /\left|\ln \left(a_{\uparrow \downarrow} / a\right)\right|} \ll \xi$. This separation of scales means that in the effective theory for fields with momenta $k \sim \sqrt{|\mu|}$ the effect of higher-momentum modes is just a local density-dependent term and one can write the energy density functional as

$$
\varepsilon\left(\psi, \psi^{*}\right)=|\nabla \psi|^{2}+\frac{8 \pi|\psi|^{4}}{\ln ^{2}\left(a_{\uparrow \downarrow} / a\right)} \ln \frac{|\psi|^{2}}{e n_{0}} .
$$

Here the complex field $\psi(\boldsymbol{\rho}, t)$ satisfies the normalization condition $N=\int|\psi(\boldsymbol{\rho}, t)|^{2} d^{2} \rho$, where $N$ is the number of particles in each component (we consider the symmetric case). The Gross-Pitaevskii equation for $\psi$ reads

$$
i \dot{\psi}=-\frac{\nabla^{2}}{2} \psi+\frac{8 \pi}{\ln ^{2}\left(a_{\uparrow \downarrow} / a\right)} \ln \left(\frac{|\psi|^{2}}{\sqrt{e} n_{0}}\right)|\psi|^{2} \psi,
$$

and the stationary one is obtained from Eq. (10) by substituting $\psi(\boldsymbol{\rho}, t)=\psi(\boldsymbol{\rho}) e^{-i \mu t}$ [for uniform liquid $\left.\mu=\mu_{0}=-4 \pi n_{0} / \ln ^{2}\left(a_{\uparrow \downarrow} / a\right)\right]$. The dimensional analysis of Eq. (10) shows that the typical length scale on which $\psi$ changes is indeed $\xi$. If $\psi$ is real and depends only on one coordinate, say $x$, this type of equation (with no explicit spatial dependence of coefficients) maps to the classical problem of a particle moving in time $x$ and coordinate $\psi$ [19]. We will discuss it in more detail in the onedimensional case. Here we mention that the surface tension (the energy per unit length of the liquid-vacuum interface) $\sigma=\int d x\left[\varepsilon(\psi, \psi *)-2 \mu_{0}|\psi|^{2}\right]=I \sqrt{\pi}\left(2 n_{0}\right)^{3 / 2} / \ln \left(a_{\uparrow \downarrow} / a\right)$, where $I=\int_{0}^{1} d z \sqrt{1-z+z \ln z} \approx 0.42$. This quantity is useful for calculating finite-size corrections to droplet's energy and the spectrum of its surface modes (see, for example, Ref. [20]). Note that such droplets with almost uniform bulk density qualitatively differ from exponentially small and dense many-body bound states of attractive twodimensional scalar bosons stabilized by the increased kinetic energy associated with their nonuniform shape [21].

We now turn to the one-dimensional case where the weakly interacting regime requires $\left|g_{\sigma \sigma^{\prime}}\right| / n \ll 1$ [22]. Strictly speaking, there is no condensate in one dimension, but it is now well understood that the energy of a weakly interacting Bose gas is correctly predicted by the Bogoliubov theory which assumes condensate [7,23]. In this way we obtain the energy density in the form of Eq. (2) where no cutoff is necessary, and the integration over momentum results in

$$
E_{1 \mathrm{D}}=\frac{1}{2} \sum_{\sigma \sigma^{\prime}} g_{\sigma \sigma^{\prime}} n_{\sigma} n_{\sigma^{\prime}}-\frac{2}{3 \pi} \sum_{ \pm} c_{ \pm}^{3}
$$

where $c_{ \pm}$are given by Eq. (3). 
Let us introduce $\delta g=g_{\uparrow \downarrow}+\sqrt{g_{\uparrow \uparrow} g_{\downarrow \downarrow}}$ and discuss the regime of repulsive intra- and attractive interspecies interactions close to the mean-field collapse instability point such that $0<\delta g \ll g=\sqrt{g_{\uparrow \uparrow} g_{\downarrow \downarrow}}$. In this regime Eq. (11) can be rewritten as

$$
\begin{aligned}
E_{1 \mathrm{D}}= & \frac{\left(g_{\uparrow \uparrow}^{1 / 2} n_{\uparrow}-g_{\downarrow \downarrow}^{1 / 2} n_{\downarrow}\right)^{2}}{2}+\frac{g \delta g\left(g_{\downarrow \downarrow}^{1 / 2} n_{\uparrow}+g_{\uparrow \uparrow}^{1 / 2} n_{\downarrow}\right)^{2}}{\left(g_{\uparrow \uparrow}+g_{\downarrow \downarrow}\right)^{2}} \\
& -\frac{2}{3 \pi}\left(g_{\uparrow \uparrow} n_{\uparrow}+g_{\downarrow \downarrow} n_{\downarrow}\right)^{3 / 2} .
\end{aligned}
$$

Similarly to the higher-dimensional cases we assume $n=n_{\uparrow}=n_{\downarrow} \sqrt{g_{\downarrow \downarrow} / g_{\uparrow \uparrow}}$. Then the structure of the energy density is $E \propto \delta g n^{2}-(g n)^{3 / 2}$ characterized by the existence of the liquid state with equilibrium density $n_{0} \sim g^{3} / \delta g^{2}$. Note that at this density $g / n \sim(\delta g / g)^{2} \ll 1$; i.e., the system is weakly interacting. Counterintuitively, liquid appears for $\delta g>0$ in the regime where the mixture is on average repulsive and where one would expect a stable gas phase. It is thus the attractive beyond-mean-field term that liquefies it.

The quantitative analysis of the droplet properties in the one-dimensional case as well as the derivation of the corresponding Gross-Pitaevskii equation goes along the same lines as in the higher-dimensional cases. In particular, in the symmetric case $g=g_{\uparrow \uparrow}=g_{\downarrow \downarrow}$ and $n=n_{\uparrow}=n_{\downarrow}$ Eq. (12) becomes

$$
E_{1 \mathrm{D}}=\delta g n^{2}-4 \sqrt{2}(g n)^{3 / 2} / 3 \pi,
$$

the equilibrium density reads

$$
n_{0}=8 g^{3} /\left(9 \pi^{2} \delta g^{2}\right)
$$

and the corresponding chemical potential equals $\mu_{0}=-\delta g n_{0} / 2$. The spinodal point is at $n=9 n_{0} / 16 \approx$ $0.56 n_{0}$. In Fig. 2 we compare the prediction of Eq. (13) valid in the limit $\delta g / g \rightarrow 0$ with our DMC results obtained also for the symmetric mixture with delta-function interactions but at finite values of $\delta g / g$. Our numerical procedure is similar to the one used in the one-dimensional one-component case [24]. The rate of convergence towards Eq. (13) indicates that the expansion of the energy continues in integer powers of $\sqrt{g / n} \propto \delta g / g$.

The Gross-Pitaevskii equation for the droplet reads

$$
i \dot{\psi}=-\psi_{x x}^{\prime \prime} / 2+\delta g|\psi|^{2} \psi-(\sqrt{2} / \pi) g^{3 / 2}|\psi| \psi,
$$

where $\psi(x, t)$ satisfies $N=\int|\psi(x, t)|^{2} d x$. It turns out that the droplet exists for any $\mu_{0}<\mu<0$ (which translates to any $N$ ) and its shape can be found analytically. For real $\psi$ Eq. (15) can be written in the form $\psi_{x x}^{\prime \prime}=-V_{\psi}^{\prime}(\psi)$, where $V(\psi)=-\delta g \psi^{4} / 2+(2 \sqrt{2} / 3 \pi) g^{3 / 2} \psi^{3}+\mu \psi^{2}$. This equation describes the trajectory of a classical particle in time

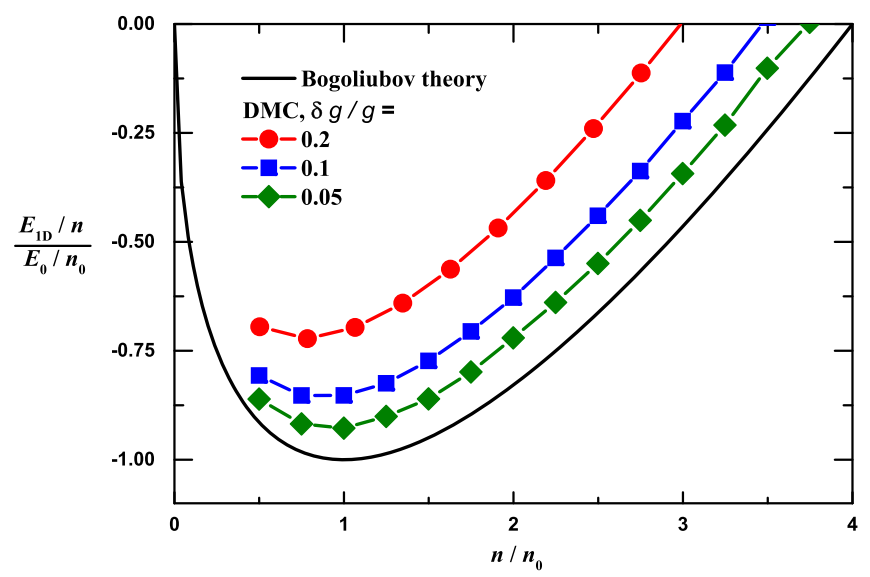

FIG. 2. The energy per particle $E_{1 \mathrm{D}} / 2 n$ versus $n$ for the symmetric one-dimensional mixture with delta-function interactions. The vertical and horizontal axes are rescaled, respectively, by $E_{0} / 2 n_{0}=\left|E_{1 \mathrm{D}}\left(n_{0}\right)\right| / 2 n_{0}$ and $n_{0}$ given from Eqs. (13)-(14). The solid black line is given by Eq. (13), exact for $\delta g / g \rightarrow 0$, and the scattered data are the DMC results for $\delta g / g=$ 0.2 (red circles), 0.1 (blue squares), and 0.05 (green diamonds).

$x$ with coordinate $\psi$ [19]. Once integrated, it reads $d \psi / \sqrt{-2 V(\psi)}=d x$. The second integration gives the shape of the droplet,

$$
\psi(x, t)=\frac{\sqrt{n_{0}} e^{-i \mu t} \mu / \mu_{0}}{1+\sqrt{1-\mu / \mu_{0}} \cosh (\sqrt{-2 \mu} x)},
$$

containing $N=2 \sqrt{n_{0} / \delta g}\left\{\ln \left[\left(1+\sqrt{\mu / \mu_{0}}\right) /\left(\sqrt{1-\mu / \mu_{0}}\right)\right]-\right.$ $\left.\sqrt{\mu / \mu_{0}}\right\}$ particles of each component. Note that in contrast to the usual single-soliton solution of the one-dimensional Schrödinger equation with attractive cubic nonlinearity [25] our droplet has a flat bulk region for $\mu \approx \mu_{0}$. We also note that in this case the typical inverse length on which $\psi$ changes is of order $\sqrt{\delta g n_{0}}$ which is much smaller than the typical momentum $\sim \sqrt{g n_{0}}$ contributing to the last (beyond-mean-field) term in Eq. (13). This justifies the low-energy theory (15). For $\mu / \mu_{0} \ll 1$ the size of the droplet increases and its density decreases with decreasing $|\mu|$. For the validity of (15) in this case we need $\mu / \mu_{0} \gg \delta g / g$ or, equivalently, $N \gg 1$.

In conclusion, weakly interacting low-dimensional Bose-Bose mixtures manifest themselves as promising candidates for studying liquid phases in the ultracold ultradilute regime and associated beyond-mean-field effects. We find that in the two-dimensional case the liquid phase is formed whenever the intraspecies interactions are repulsive and the interspecies one is attractive. This differs from the three-dimensional case where $\left|g_{\uparrow \downarrow}\right|$ should be larger than $\sqrt{g_{\uparrow \uparrow} g_{\downarrow \downarrow}}$. Remarkably, the one-dimensional mixture liquefies for $\left|g_{\uparrow \downarrow}\right|<\sqrt{g_{\uparrow \uparrow} g_{\downarrow \downarrow}}$; this effect is completely missed by the mean-field approximation. Interestingly, one-dimensional droplets can be described 
analytically and it is tempting to study their dynamical and transport properties. In any dimension the almost complete cancellation of the first-order terms in the energy functional of the liquid gives one an opportunity to test higher-order terms and their universality. It is then relevant, although theoretically challenging, to go beyond the Bogoliubov approximation as it has been done in the scalar twodimensional case [8].

We acknowledge support by the IFRAF Institute. The research leading to these results received funding from the European Research Council (FR7/2007-2013 Grant Agreement No. 341197) and the MICINN (Spain) Grant No. FIS2014-56257-C2-1-P. The Barcelona Supercomputing Center (The Spanish National Supercomputing CenterCentro Nacional de Supercomputación) is acknowledged for the provided computational facilities.

[1] H. Kadau, M. Schmitt, M. Wenzel, C. Wink, T. Maier, I. Ferrier-Barbut, and T. Pfau, Observing the Rosensweig instability of a quantum ferrofluid, Nature (London) $\mathbf{5 3 0}$, 194 (2016).

[2] I. Ferrier-Barbut, H. Kadau, M. Schmitt, M. Wenzel, and T. Pfau, Observation of Quantum Droplets in a Strongly Dipolar Bose Gas, Phys. Rev. Lett. 116, 215301 (2016).

[3] D. S. Petrov, Quantum Mechanical Stabilization of a Collapsing Bose-Bose Mixture, Phys. Rev. Lett. 115, 155302 (2015).

[4] F. Wächtler and L. Santos, Quantum filaments in dipolar Bose-Einstein condensates, Phys. Rev. A 93, 061603(R) (2016).

[5] H. Saito, Path-integral Monte Carlo study on a droplet of a dipolar Bose-Einstein condensate stabilized by quantum fluctuation, J. Phys. Soc. Jpn. 85, 053001 (2016).

[6] In particular, theories of a scalar two-dimensional Bose gas are quite involved as they have to take into account beyondmean-field effects, see M. Schick, Two-dimensional system of hard-core bosons, Phys. Rev. A 3, 1067 (1971); Ref. [7]; Yu. E. Lozovik and V. I. Yudson, On the ground state of the two-dimensional non-ideal Bose gas, Physica (Amsterdam) 93A, 493 (1978); A. Yu. Cherny and A. A. Shanenko, Dilute Bose gas in two dimensions: Density expansions and the Gross-Pitaevskii equation, Phys. Rev. E 64, 027105 (2001); C. Mora and Y. Castin, Extension of Bogoliubov theory to quasicondensates, Phys. Rev. A 67, 053615 (2003); Ref. [8]; L. Pricoupenko, Implicit ladder summation in the HartreeFock-Bogoliubov approach, Phys. Rev. A 84, 053602 (2011).

[7] V. N. Popov, To the theory of superfluidity of the twodimensional and one-dimensional Bose systems, Teor. Mat. Fiz. 11, 354 (1971) [Theor. Math. Phys. 11, 565 (1972)].

[8] C. Mora and Y. Castin, Ground State Energy of the TwoDimensional Weakly Interacting Bose Gas: First Correction Beyond Bogoliubov Theory, Phys. Rev. Lett. 102, 180404 (2009).
[9] S. T. Beliaev, Application of the methods of quantum field theory to a system of bosons, Zh. Eksp. Teor. Fiz. 34, 417 (1958) [Sov. Phys. JETP 7, 289 (1958)].

[10] E. M. Lifshitz and L. P. Pitaevskii, Statistical Physics, Part 2 (Pergamon Press, Oxford, 1980).

[11] R. Jackiw, Delta-function potentials in two- and threedimensional quantum mechanics in M.A.B. Bég Memorial Volume, edited by A. Ali and P. Hoodbhoy (World Scientific, Singapore 1991).

[12] S. Pilati, J. Boronat, J. Casulleras, and S. Giorgini, Quantum Monte Carlo simulation of a two-dimensional Bose gas, Phys. Rev. A 71, 023605 (2005).

[13] L. Reatto and G. V. Chester, Phonons and the properties of a Bose System, Phys. Rev. 155, 88 (1967).

[14] See Supplemental Material at http://link.aps.org/ supplemental/10.1103/PhysRevLett.117.100401 for more details on our DMC procedure, on the choice of the guiding wave function, on finite-size and finite-range corrections.

[15] The negative-pressure state that we observe for $n<n_{0}$ is actually the ground state for our finite-size system (phase separation is prevented by the surface tension). The energy of the metastable branch can be calculated down to the spinodal point, see J. Boronat, J. Casulleras, and J. Navarro, Monte Carlo calculations for liquid ${ }^{4} \mathrm{He}$ at negative pressure, Phys. Rev. B 50, 3427 (1994).

[16] D. S. Petrov and G. V. Shlyapnikov, Interatomic collisions in a tightly confined Bose gas, Phys. Rev. A 64, 012706 (2001).

[17] C. D'Errico, M. Zaccanti, M. Fattori, G. Roati, M. Inguscio, G. Modugno, and A. Simoni, Feshbach resonances in ultracold ${ }^{39}$ K, New J. Phys. 9, 223 (2007).

[18] M. Lysebo and L. Veseth, Feshbach resonances and transition rates for cold homonuclear collisions between ${ }^{39} \mathrm{~K}$ and ${ }^{41} \mathrm{~K}$ atoms, Phys. Rev. A 81, 032702 (2010).

[19] J. Goldstone and R. Jackiw, Quantization of nonlinear waves, Phys. Rev. D 11, 1486 (1975); N. Christ and T. D. Lee, Quantum expansion of soliton solutions, ibid. 12, 1606 (1975).

[20] A. Bulgac, Dilute Quantum Droplets, Phys. Rev. Lett. 89, 050402 (2002).

[21] H.-W. Hammer and D. T. Son, Universal Properties of TwoDimensional Boson Droplets, Phys. Rev. Lett. 93, 250408 (2004).

[22] For an atomic mixture under the quasi-one-dimensional confinement characterized by the oscillator length $l_{0}$ one has $g_{\sigma \sigma} \approx a_{\sigma \sigma^{\prime}}^{(3 \mathrm{D})} / 2 l_{0}^{2}$, see M. Olshanii, Atomic Scattering in the Presence of an External Confinement and a Gas of Impenetrable Bosons, Phys. Rev. Lett. 81, 938 (1998).

[23] E. H. Lieb and W. Liniger, Exact analysis of an interacting Bose gas. I. The general solution and the ground state, Phys. Rev. 130, 1605 (1963).

[24] G. E. Astrakharchik and S. Giorgini, Correlation functions and momentum distribution of one-dimensional Bose systems, Phys. Rev. A 68, 031602 (2003).

[25] V.E. Zakharov and A. B. Shabat, Exact theory of two-dimensional self-focusing and one-dimensional selfmodulation of waves in nonlinear media, Zh. Eksp. Teor. Fiz. 61, 118 (1971) [Sov. Phys. JETP 34, 62 (1972)]. 